\title{
Vita brevis of antibubbles
}

\author{
Stéphane Dorbolo ', Nicolas Vandewalle ', Etienne Reyssat ${ }^{2}$ and David Quéré ${ }^{2}$ \\ GRASP, Université de Liège • Belgium \\ PMMH, UMR 7636 du CNRS, ESPCI, Paris • France
}

\begin{abstract}
Starting here in the Latin world, a bubble is originally a bulla, that is, a ball; a metallic ball, first, attached to a seal, and by continuity the papal decree stamped with this seal; or a bulla, blown by children from a soapy water, or observed inside a sparkling liquid. Nowadays, bolla designates in Italian both the bubble inside a liquid and the papal seal - in French, we similarly have bulle for both meanings, as in Spanish with bula (where bubbles in liquids are however most often qualified by the more modern burbuja). Hence a bubble is first defined by its shape, that is, the pure sphere that we all observed for small gas cavities in Champagne, or for larger spherical bubbles blown by children, the pretext for admirable paintings by Chardin or Manet. Here we describe the negative of this object: as an antibolla in Italian (or antibulle in French [1], and antibula in Spanish) designated the bolla of an antipope (when they existed), an antibubble will be the contrary of a soap bubble, namely, a thin shell of air surrounding water, and immersed in water [2]. We first discuss the way to generate such objects; then, we comment on their (brief) life and their death, comparing them with those of soap bubbles.
\end{abstract}

\section{Antibubble generators}

The simplest method to obtain an antibubble consists of pouring soapy water inside a bath of the same nature (Fig. 1). The bath is first filled, in such a way that the surface is clean, without any bubble. Then, a drop of the same liquid is deposited at this surface. Owing to the presence of surfactants, it floats a few seconds before merging [3]. This situation can be made much longer by making the bath vibrate, as recently shown by Couder et al. [4]. But even without this trick, the floating drop can be gently fed so that it becomes centimetric. Pushing this globule below the surface with a jet can produce an antibubble. An even simpler way consists of making the jet directly impact the bath, with a velocity large

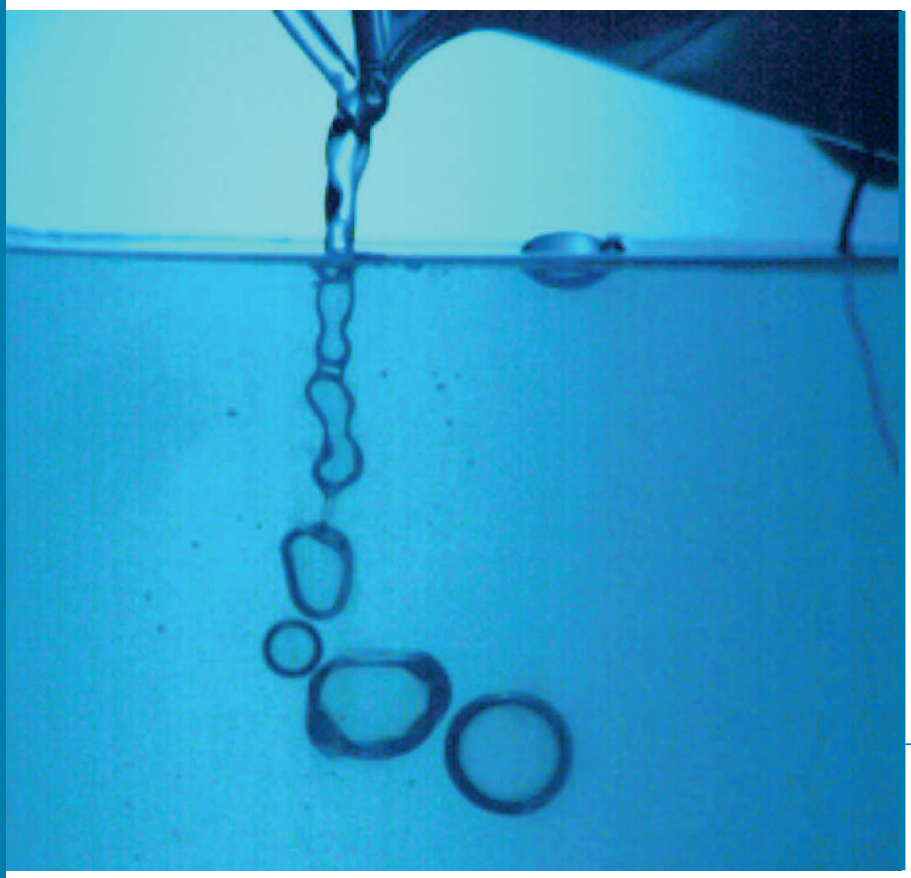

enough to make it penetrate the bath coated with a film of air. The Rayleigh-Plateau instability (which transforms a jet coming out of a faucet into drops) may destabilize this jet, which generates a whole flottilla of antibubbles (Fig. 1).

The presence of a shell of air will make the antibubble rise, pushed by buoyancy forces. The velocity of rise allows us to determine the typical thickness of the shell, which is found to be of the order of 5 microns, a value comparable to the thickness of a soap bubble [5]. In order to avoid this rise (which is fatal for our globule, as it reaches the upper surface of the bath), the impinging liquid can be salted, and the antibubble formed in a bath containing (heavy) glycerol at the bottom: then, the antibubble will first sink, and then stop at the place which matches its density. There it can be peacefully observed.

Such an antibubble will be centimetric. It is shining due to a total reflection of the light on the film of air (Fig. 2a). The shape is spherical (which minimizes the surface area of the interfaces), but an air bubble of the same volume as the air shell would of course have a much smaller surface area: as for a soap bubble, our object will thus be only metastable or unstable. You can also observe a slight bump at the top: air tends to rise and to accumulate at this pole. Interference fringes indicate variations of the film thickness. All these observations show that the film of air gets thinner at the bottom, which is confirmed by the motion of the fringes, and by the fact that the antibubble generally starts bursting close to the South pole.

\section{Drainage}

The lifetime of an antibubble is generally several minutes [5]. Indeed, it does not suffer the main cause of aging for a classical soap bubble, namely, evaporation, but like it, it is subjected to drainage. Air slowly rises along the shell (or in other words, the inner globule slowly sinks in the film of air on which it sits). The kinetics of drainage should be dictated by a balance between viscous friction (in the film) and buoyancy. Denoting as $\rho$ the water density, $g$ the gravity acceleration, $\eta$ the air viscosity and $\varepsilon$ the film thickness, this balance can be written dimensionally: $\eta V / \varepsilon^{2} \sim \rho g$, where $V$ is the mean velocity of the rising air. This velocity is all the smaller since the film is thin, owing to the boundary condition at the air/water interface, which should nearly stop the flow at this interface (the water viscosity being about 100 times larger than the air viscosity): the thinner the film, the larger the proportion of gas governed by this boundary condition, and thus the slower the motion. This kinetics (often called a Reynolds kinetics) was also observed by Couder, who studied the thinning of an air film placed between a bath of viscous oil and a large oil drop deposited on this bath [4]. If an antibubble bursts when it reaches a thickness $\varepsilon_{o}$, the kinetics of thinning will be dominated by the approach to $\varepsilon_{0}$. Since the flow takes place on the scale $R$ of the globule, we deduce that the drainage time should scale as $\tau \sim R / V\left(\varepsilon_{0}\right) \sim \eta R / \rho g \varepsilon_{0}{ }^{2}$.

The question is thus to determine $\varepsilon_{0}$. For "classical" bubbles, and if the surfactants are charged, the final film thickness results from a

4 Fig. 1: Submarine photo showing the generation of antibubbles from an impacting jet. The black contour, around each globule, betrays the existence of a film of air. 
balance between van der Waals forces (which tend to thin the film) and a repulsion of electrostatic origin: the counter-ions coming from the surfactant heads occupy a certain zone, which (entropically) resists squeezing. These (meta)stable films of typical thickness a few tens of nanometers are the so-called black films (observed by Newton, Hooke and Jean Perrin). For antibubbles, the situation should be quite different (Figure 2b): van der Waals forces thin the film without anything to stop the thinning process. Hence the thickness $\varepsilon_{o}$ should be given by the range of these forces, that is, about 100 nanometers. This gives for the lifetime $\tau$ about one hour, at most.

The antibubbles are generally observe to live shorter (less than 10 minutes). This quicker aging might be related to the formation of radial channels: instead of having a homogeneous flow of air along the shell, air seems to be transported from the film to the channels (thus, over a short distance, which is the inter-channel distance); then, it quickly rises along the channels (as water does in gutters, or in Plateau boarders, that is, the channels separating adjacent films in a foam). The distance $L$ between these channels is millimetric, which makes $\tau \sim L / V\left(\varepsilon_{\mathrm{o}}\right)$ about ten times smaller than when assuming a homogeneous flow, in agreement with the observations (lifetime of a few minutes instead of one hour).

\section{Death of the antibubble}

The bursting of an antibubble can be captured using a high speed camera. Figure 3 shows such an event. As mentioned earlier, the explosion often starts at the South pole where the film is thinner. However, the most remarkable point is the fact that the air film disaggregates at several places at the same time. This mode of explosion resembles the so-called spinodal dewetting of nanometric liquid films [6]: without any stabilizing forces for the film, holes result from the amplification of thermal fluctuations, owing to van der Waals forces. Several holes are thus likely to open simultaneously, with a spatial distribution that integrates the (destabilizing) action of van der Waals forces, and the (stabilizing) effect of surface tension, which opposes surface fluctuations.

The mode of rupture observed in Figure 3 thus confirms that the film thinned down to the van der Waals thickness. In contrast with bubbles, nothing then stabilizes the globule. You will notice in the same Figure a bulge at the North pole, resulting from the drainage of air, which accumulated at this place.

\section{Conclusion}

In contrast to soap bubbles, antibubbles are globules which are intrinsically unstable, without the electrostatic interaction making possible the existence of metastable black films: there is no black antibubble. Their lifetime thus simply results from the slow rise of

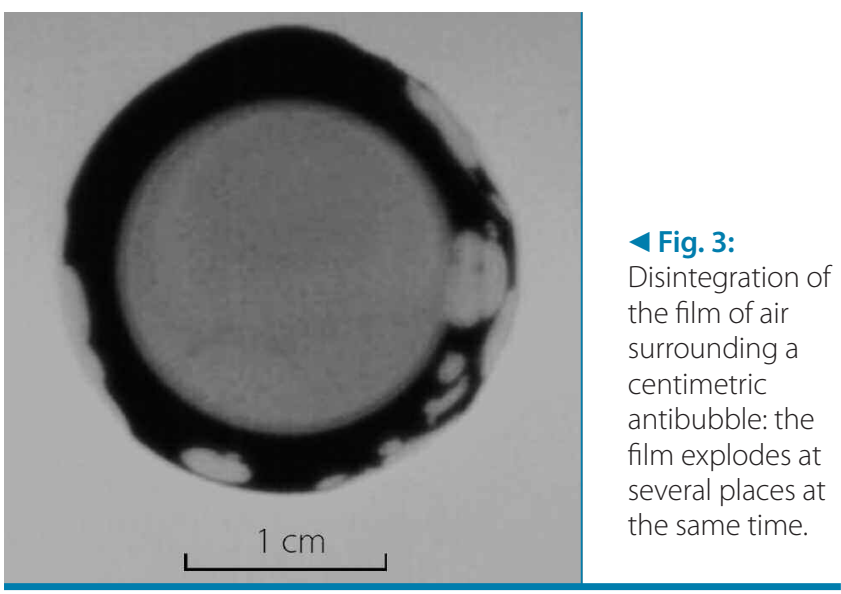

air within the shell. The thinning takes place until the film thickness becomes of the order of the range of van der Waals forces, around $100 \mathrm{~nm}$. Then, van der Waals forces (which make the outer water attract the inner water) squeeze the shell, provoking its explosion.

The slowness of the drainage (several minutes) makes the antibubble an interesting object for the encapsulation of chemicals or colloidal materials, which can thus be transported without mixing nor coalescence in microfluidic devices. The content can be recovered by activating the film bursting, owing to a pulse of pressure, or by any means favouring the film rupture. The dynamics of the rupture would itself deserve a study; unlike bubbles, for which the kinetics of the explosion is dictated by the film properties (density or viscosity), the bursting speed should be limited by the properties of the surrounding liquid (the film of air having a negligible density and viscosity). Note finally that a special case of antibubble is the one where the film consists of oil [7], making this film much more viscous. In addition, the density contrast between the two fluids is smaller in this case, making this variety of antibubble much more resistant to aging.

\section{References}

[1] E. Littré, Dictionnaire de la langue Française, Hachette (1873).

[2] W. Hughes and A.R. Hughes, Nature 129, 59 (1932) ; C.L. Stong, Scientific American 230, 116 (1974).

[3] Y. Amarouchène, G. Cristobal and H. Kellay, Physical Review Letters 87, 206104 (2001).

[4] Y. Couder, E. Fort, C.H. Gautier and A. Boudaoud, Physical Review Letters 94, 177801 (2005).

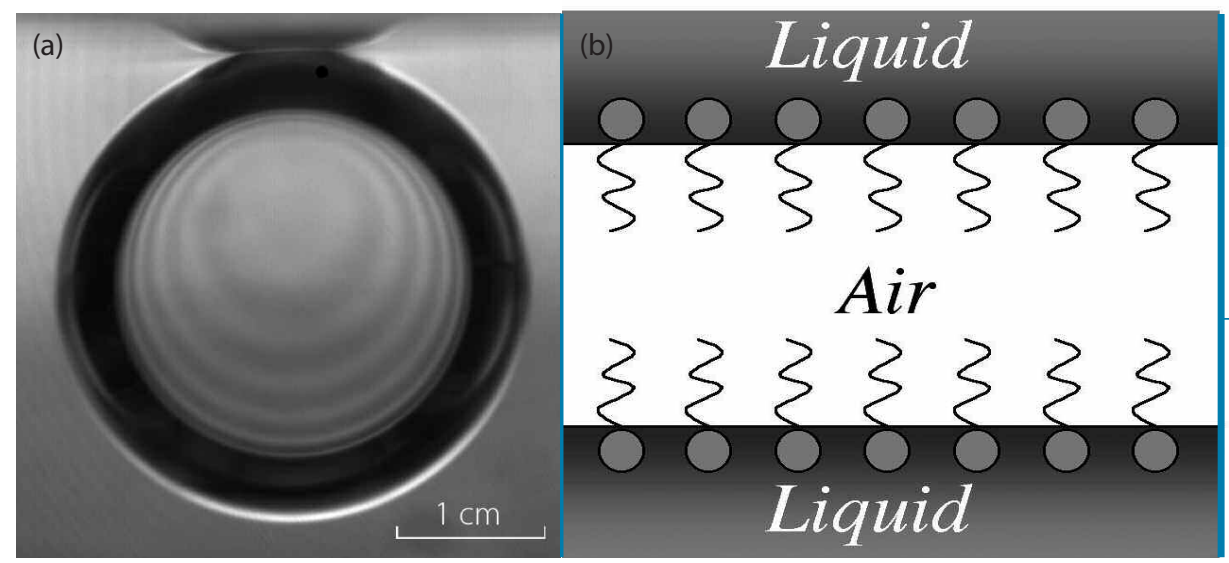

[5] S. Dorbolo, E. Reyssat, N. Vandewalle and D. Quéré, Europhysics Letters 69,966 (2005).

[6] G. Reiter, Science 282, 888 (1998).

[7] K.P. Galvin, S.J. Pratten, G.M. Evans and S. Biggs, Langmuir 22, 52 (2006).

4 Fig. 2: (a) Photo of an antibubble close to the surface of the bath. (b) Transverse cut of the air film: the surface is coated by surfactant molecules, whose hydrophobic tails face each other. 